\title{
Generalization of the Dissimilarity Measure of Fuzzy Sets
}

\author{
Faramarz Faghihi \\ Bioinformatics Laboratory, Nanobiotechnology Research Center \\ Avesina Research Institute, ACECR, Tehran, Iran \\ faghihi@avesina.ac.ir
}

\begin{abstract}
Fuzzy sets theory has several applications in biomedicine. The dissimilarity of fuzzy sets is used for this purpose. In this paper, we present the generalized equation of the dissimilarity of fuzzy sets. This relation is based on the set theoretic point of view. The parameter of this generalized equation and also its relation to a recently introduced metric defined in fuzzy hypercube are discussed.
\end{abstract}

Mathematics Subject Classification: 03E72

Keywords: fuzzy sets, dissimilarity of fuzzy sets, NTV metric, fuzzy hypercube

\section{Preliminary Notes}

Fuzzy sets theory is the extension of classical sets theory [8]. A fuzzy set is defined as follows.

Let $X$ be a set, $X=\left\{x_{1}, x_{2}, \ldots, x_{n}\right\}$

a fuzzy set in $X$ is given by 
$A=\left\{\left(x, \mu_{A}(x)\right) \mid x \in X\right\}, \quad \mu_{A}(x) \in[0,1]$.

The membership function $\mu_{A}(x) \in[0,1]$ is such that $\mu_{A}(x)$ quantifies the grade of membership of the element ${ }^{x}$ of $X$. The cardinality of a fuzzy set is defined as $\left|\mu_{A}\right|=\sum_{x \in X} \mu_{A}(x)$.

Therefore, we can define a set as an $n$-dimensional ordered fuzzy set $P=\left(\mu\left(x_{1}\right), \mu\left(x_{2}\right), \ldots, \mu\left(x_{n}\right)\right)$

For further study on fuzzy sets theory, we refer to [4].

The fuzzy hypercube is a geometrical interpretation of fuzzy sets [2].

For a set $X=\left\{x_{1}, x_{2}, \ldots, x_{n}\right\}$, a fuzzy subset is just a mapping ${ }^{\mu}$ : $X \rightarrow[0,1]$, The unit hypercube is constructed as the set of all possible fuzzy subsets of $X$ and it is $I^{n}=[0,1]^{n}$,

Thus, any fuzzy subset $\mu$ determines a point $P=\left(\mu\left(x_{1}\right), \mu\left(x_{2}\right), \ldots, \mu\left(x_{n}\right)\right)$ in the unit hypercube $I^{n}, P \in I^{n}$

Reciprocally, any point $A=\left(a_{1}, \ldots, a_{n}\right) \in I^{n}$ generates a fuzzy subset $\mu$ defined by $\mu\left(x_{i}\right)=a_{i}, \quad i=1, \ldots, n$

Non fuzzy or crisp subsets of $\mathrm{X}$ are given by mappings $I^{n}=\{0,1\}^{n}$, and are located at the $2^{n}$ corners of the n-dimensional unit hypercube $I^{n}$.

\section{Generalization of the dissimilarity of fuzzy sets}

To represent DNA and RNA sequences as ordered fuzzy sets and so points in fuzzy hypercube and to compare their similarities and dissimilarities, the Fuzzy Polynucleotide Space was introduced in [6]. The distance they defined was based on the concept of fuzzy Hamming distance and the fuzzy entropy theorem [4, 2] and it has been shown that it is indeed a metric $[3,1]$. 
Given two ordered fuzzy sets, $P=\left(p_{1}, p_{2}, \ldots, p_{n}\right)$ and $Q=\left(q_{1}, q_{2}, \ldots, q_{n}\right)$ in the unit hypercube $I^{n}$, not both equal to the empty set, the distance between $P$ and $Q$ is given by

$$
d(P, Q)=\frac{\sum_{i=1}^{n}\left|p_{i}-q_{i}\right|}{\sum_{i=1}^{n} \max \left\{p_{i}, q_{i}\right\}} .
$$

Which has been called 'NTV metric' [1]. We show that such defined distance measure is indeed the dissimilarity measure of two fuzzy sets and also is one case of a general relation for dissimilarity of fuzzy sets.

For two fuzzy sets, $P$ and $Q$,

$P=\left(p_{1}, p_{2}, \ldots, p_{n}\right), \quad Q=\left(q_{1}, q_{2}, \ldots, q_{n}\right) \quad \in I^{n}$

it is defined the fuzzy operator $\wedge$ (AND) as

$R=P \wedge Q, R=\left(r_{1}, \ldots, r_{n}\right), r_{i}=p_{i} \wedge q_{i}=\min \left\{p_{i}, q_{i}\right\}$,

The fuzzy operator $\vee(\mathrm{OR})$ is defined as:

$R=P \vee Q, R=\left(r_{1}, \ldots, r_{n}\right), r_{i}=p_{i} \vee q_{i}=\max \left\{p_{i}, q_{i}\right\}$,

Werners [7] presented a general definition for operators of $\wedge$ and $\vee$ depending of a $\partial$ parameter as

$$
\begin{aligned}
& p_{i} \wedge q_{i}=\partial \min \left\{p_{i}, q_{i}\right\}+(1-\partial) \frac{\left(p_{i}+q_{i}\right)}{2}, \\
& p_{i} \vee q_{i}=\partial \max \left\{p_{i}, q_{i}\right\}+(1-\partial) \frac{\left(p_{i}+q_{i}\right)}{2},
\end{aligned}
$$

For the fuzzy sets $P$ and $Q$, we consider a set-theoretic operation based similarity measure [5]. That is defined as

$$
\text { Simil }(P, Q)=\frac{P \cap Q}{P \bigcup Q}
$$

Where $\cap$ and $U$ are intersection and union of fuzzy sets respectively.

We can consider the operators of $\wedge$ and $\vee$ for intersection and union of $P$ and $Q$ respectively.

$$
\operatorname{Simil}(P, Q)=\frac{|P \wedge Q|}{|P \vee Q|} \quad:[0,1]
$$


Using the relations (2), (3)

$$
\operatorname{Simil}(P, Q)=\frac{P \wedge Q}{P \vee Q}=\frac{2 \partial \sum_{i=1}^{n} \min \left(p_{i}, q_{i}\right)+(1-\partial) \sum_{i=1}^{n}\left(p_{i}, q_{i}\right)}{2 \partial \sum_{i=1}^{n} \max \left(p_{i}, q_{i}\right)+(1-\partial) \sum_{i=1}^{n}\left(p_{i}, q_{i}\right)}
$$

Using the relation

$$
\sum_{i=1}^{n}\left|p_{i}-q_{i}\right|=\left(\sum_{i=1}^{n} \max \left(p_{i}, q_{i}\right)-\sum_{i=1}^{n} \min \left(p_{i}, q_{i}\right)\right)
$$

and

$$
\operatorname{Dissimil}(P, Q)=1-\operatorname{Simil}(P, Q)
$$

we have

$$
\operatorname{Dissimil}(P, Q)=\frac{2 \partial\left(\sum_{i=1}^{n}\left|p_{i}-q_{i}\right|\right)}{2 \partial \sum_{i=1}^{n} \max \left(p_{i}, q_{i}\right)+(1-\partial) \sum_{i=1}^{n}\left(p_{i}+q_{i}\right)}
$$

The equation (8) can be considered as the generalization of the dissimilarity of fuzzy sets.

' $\partial$ parameter' determines how much operators of $\wedge$ and $\vee$ are considered to be uncertain. For $\partial=1$, we obtain 'NTV metric'.

$\partial=1$ means that operators are completely crisp but $\partial=0$ means that operators are completely uncertain. In such case, there is no difference between Min and Max operators. Therefore, every pair of fuzzy sets will have a similarity value as 1 (100\% similarity). It means that in this case, the difference between any pairs of fuzzy sets tends to zero.

Although $\partial=1$ seems a good choice but some questions remain to be answered. What is the geometric meaning of $\partial$ parameter? What is the application of different values of $\partial$ parameter in solving problems? Does the relation (8) with all values of $\partial$ parameter present a metric? 


\section{Conclusion}

We obtained the generalized relation for the dissimilarity of fuzzy sets and showed that this equation results in 'NTV metric' as $\partial=1$.

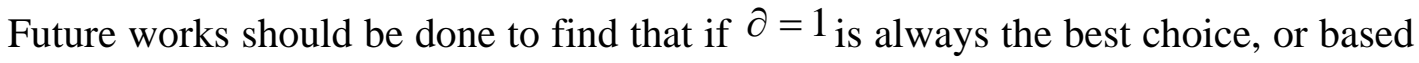
on the fuzzy sets theoretic meaning of ' $\partial$ parameter' different values of it should be applied in different applications. Another open problem is to investigate that if such generalized relation could always be a metric or not.

\section{References}

[1] A. Dress, T. Lokot, A simple proof of the triangle inequality for the NTV metric, Appl. Math. Lett, 16 (2003), 809-813.

[2] B. Kosko, Neural networks and fuzzy systems. Prentice-Hall, Englewood Cliffs, NJ, 1992.

[3] J.J. Nieto, A. Torres, M.M. Vazquez-Trasande, A metric space to study differences between polynucleotides. Appl. Math. Lett, 27 (2003), 81-101.

[4] K. Sadegh-Zadeh, Fuzzy genomes, Artificial Intelligence in Medicine, 18 (2000), 1-28.

[5] M. Setnes, R. Babuska, U. Kaymak U, H.R.N. Lemke, Similarity Measures in Fuzzy Rule Base Simplification. IEEE Trans on Systems Man and Cybernetics, 28 (1998), 376-386.

[6] A. Torres, J.J. Nieto, The fuzzy polynucleotide space: basic properties, Bioinformatics, 19 (2003), 587-592. 
[7] B.M. Werners, An Interactive Fuzzy Programming System, Fuzzy Sets and Systems, 23 (1987), 131-147.

[8] L.A. Zadeh, Fuzzy Sets, Information and Control, 8 (1965), 338-352.

Received: July 27, 2007 